\title{
UMA ANÁLISE CRÍTICA DO PAPEL E IMPORTÂNCIA SOCIOECONÔMICA DAS INDICAÇÕES GEOGRÁFICAS EM PAÍSES EM DESENVOLVIMENTO
}

\author{
Mateus Manfrin Artêncio, Janaina de Moura Engracia Giraldi ${ }^{1}$, \\ Simone Vasconcelos Ribeiro Galina \\ Faculdade de Economia, Administração e Contabilidade de Ribeirão Preto - FEA-RP/USP, São Paulo, (Brasil).
}

\section{DETALHES DO ARTIGO}

\section{Histórico do Artigo:}

Recebido em: 10 de dezembro de 2018

Aceito: 08 de junho de 2019

Disponível online: 01 de agosto de 2019

Sistema de revisão "Double blind review"

\section{Editor Científico}

Ilan Avrichir

\section{Palavras-chaves:}

Informação de Origem

Indicação Geográfica

Países em Desenvolvimento

\section{RESUMO}

O artigo propõe compreender o papel e importância das indicações geográficas (IGs) em países em desenvolvimento ou emergentes, devido à sua potencialidade na melhora de índices socioeconômicos locais, através da proteção e valorização do produto, assim como de sua região produtora. Presente principalmente no segmento de alimentos, o conceito de IG é utilizado para identificar e sinalizar a origem de um produto ou serviço, quando seu local de produção tenha se tornado conhecido por isso ou quando certa característica ou qualidade do bem se deva à sua origem geográfica. Para tanto, foram analisados trabalhos acadêmicos que abordassem a implementação e gestão de IGs em países com diferentes níveis de desenvolvimento, buscados nas bases de dados Scopus e Web of Science. A pesquisa é exploratória e bibliográfica, tendo como base a leitura crítica de 40 artigos. Desses, grande parte explora o processo e desafios envolvidos na implementação de indicações geográficas em diversos contextos e produtos. Outros, analisam a questão estrutural interna e planejamento de estratégias das indicações geográficas. Já outra parcela mensura e analisa a imagem que as IGs transmitem, seu papel como sinalizadoras de procedência e a influência que exercem sobre os consumidores. A partir desta análise, verificou-se que nenhum dos artigos relaciona ou examina especificamente a questão do uso da indicação geográfica como ferramenta de alavancagem de desenvolvimento para países/regiões em desenvolvimento, encontrando-se uma lacuna no arcabouço teórico da área e a necessidade de estudos e casos práticos que comprovem essa relação entre a utilização de IG e desenvolvimento local.

\section{Introdução}

Com o aumento da população mundial, diminuição de fronteiras econômicas entre países e a globalização, a necessidade de comércio de alimentos aumentou exponencialmente, o que faz com que os produtos agrícolas sejam produzidos e escoados em escala, ou seja, um processo de comoditização para atender a demanda mundial. Em contrapartida, uma vertente crescente de consumidores com preferências a alimentos oriundos de processos produtivos socioambientalmente corretos, que se traduzam em saúde e qualidade, traz novas oportunidades e desafios para os fornecedores da cadeia alimentícia (Yeung \& Kerr, 2011; Zenker, 2015).
Em vista disso, alguns produtores têm se preocupado com fatores de produção que antes eram negligenciados, no intuito de diferenciar seu produto e atender as necessidades de consumidores mais individualizados e conscientes. De modo a alcançar essa distinção, agregação de valor e preços de venda mais altos, busca-se a valorização do local/território através do realce das características que possam influenciar o produto de maneira positiva, como as particularidades naturais do local e da produção, por meio do registro de indicação geográfica (IG). Conforme o Acordo sobre Aspectos dos Direitos de Propriedade Intelectual Relacionados ao Comércio (TRIPs - do inglês Agreement on TradeRelated Aspects of Intellectual Property Rights), as IGs são definidas como indicações que identificam um produto como originário de uma região ou local

\footnotetext{
${ }^{1}$ Contato do autor - Email: igiraldi@usp.br
} 
situado no território de um de seus países-membros, cujas características naturais ou sociais atribuem a esse produto qualidade, reputação ou outra característica que o distingue do restante de sua categoria (WIPO, 2019). As IGs são um tipo de propriedade intelectual, protegidas sob uma série de ferramentas legais (Charters, Spielmann \& Babin, 2017). Logo, através do registro de indicação geográfica, pode-se atrelar o produto à região em que foi produzido, consequentemente aos fatores naturais e humanos desta, designando ao mesmo uma identidade própria e maior confiabilidade, ou seja, diferenciando-o dos similares através de sua região de origem (Chamorro, Rubio \& Miranda, 2015; Dinnie, 2016).

A IG é responsável pela representação e sinalização dos atributos ímpares do local e modo de produção, o que a confere o papel de agente protetora e promovedora tanto do produto quanto de sua região (Fonzo \& Russo, 2015). Assim, as indicações geográficas possibilitam a sinalização da qualidade de produtos e, consequentemente, o acesso de produtores a nichos de mercado específicos (Elango \& Wieland, 2017). Por meio da força coletiva, as IGs proporcionam ainda oportunidades para que produtores aprimorem seus processos produtivos e estabeleçam diferencial competitivo. Em posse desse conhecimento, países desenvolvidos as utilizam e se beneficiam de suas vantagens há algum tempo, possuindo cerca de $90 \%$ dos registros das mais de $10 \mathrm{mil}$ indicações geográficas espalhadas pelo mundo, em sua maioria no setor agroalimentar (Mascarenhas \& Wilkinson, 2014).

Por outro lado, mesmo com o grande potencial e riqueza natural da maioria dos países em desenvolvimento, e a possibilidade de explorá-los de forma sustentável e agregadora através das indicações geográficas, ainda são poucos os registros desse tipo de propriedade intelectual nesses locais (Wilkinson, Cerdan \& Dorigon, 2017).

Tal fato é explicado por Mascarenhas e Wilkinson (2014) como consequência da falta de estrutura institucional, desconhecimento dos produtores ou da ineficiência do ambiente político-legal, que falha ao não estruturar e organizar a possibilidade apropriadamente.

Tendo em vista o paralelo entre as vantagens e possibilidades que as IGs trazem para as regiões e a oportunidade que os países em desenvolvimento têm de utilizá-las para gerar progresso em seus mercados doméstico e internacional, indaga-se: "O que foi produzido em termos de pesquisa acadêmica sobre as indicações geográficas de países em desenvolvimento?".

Dessa forma, o objetivo deste artigo é verificar a publicação de artigos sobre IGs com origem em países em desenvolvimento. Os objetivos específicos consistem em: a) entender o desenvolvimento e contexto atual das IGs no mundo; b) compreender seus impactos positivos e negativos; c) identificar os desafios que os países em desenvolvimento enfrentam ao implementá-las; d) sugerir possíveis melhorias para seu uso e gestão nesses ambientes.

Diante da importância e inovação do tema, principalmente no caso dos países em desenvolvimento, uma série de trabalhos lidam com questões relacionadas às IGs, porém não há uma revisão geral e que relacione os resultados desses artigos. Afora reunir e analisar os artigos sobre indicação geográfica, é importante também evidenciar a escassez de estudos de IGs em países em desenvolvimento, uma vez que as IGs se mostram ferramentas capazes de mudar os índices socioeconômicos da região que representam (Grunert \& Aachmann, 2016).

Ademais, a IG é uma vantagem competitiva que pode ser construída e sinalizada para mercados externos, ou seja, um ativo potencial para incorporar valor ao produto e exportá-lo (Alonso, 2017; Castro \& Giraldi, 2018). Entende-se também que os investimentos em IG e seu uso como certificação possam ser um dos caminhos para assegurar o consumidor e aumentar sua confiança a respeito do produto, o que seria interessante para aprimorar a percepção do mercado acerca das produções dos países emergentes.

Para muitos dos países emergentes, cujos setores e IGs contam com o apoio de órgãos e ações governamentais de apoio, os resultados podem auxiliar no direcionamento de esforços e investimentos dessas instituições, como por exemplo, para a formação da identidade, imagem e propagação das IGs como marcas; o alinhamento entre produtores e cooperativas; a constituição de leis e diretrizes para a implementação e manutenção das IGs; e, principalmente, o reconhecimento e conscientização de consumidores e produtores desses países a respeito das IGs. Dessa forma, as recomendações de estratégias aconselhadas ao uso e 
Uma Análise Crítica do Papel e Importância Socioeconômica das Indicações Geográficas em Países em Desenvolvimento

gestão de IGs em países em desenvolvimento apresentam a relevância prática do artigo.

Isto posto, a revisão de literatura a seguir é apresentada para melhor entendimento dos temas, além da forma como têm sido explorados.

\section{Revisão de Literatura}

\section{Vantagens e benefícios obtidos no uso de indicações geográficas}

Criada em meados do século XVIII, para delimitar a produção de vinho da região do Porto, em Portugal, o uso da origem para proteção da produção regional se tornou um fenômeno bastante comum na Europa, se expandindo para outros países e produtos e atribuindo reconhecimento às respectivas regiões produtoras. Como exemplo, podem-se citar as comunas italianas de Parma e Gorgonzola, pela produção de presunto e queijo, respectivamente; ou a região de produção de vinho espumante, Champagne-Ardene, na França; a tequila do México; ou o chá preto da cidade de Darjeeling, na Índia (Duguid, 2013; Viot \& Passebois-Ducros, 2010).

Amiúde, esses e outros produtos passaram a ser sinônimo de categorias através de sua origem que, sendo usada a princípio para proteção, suscita diferenciação e agregação de valor, modificando o consumo dos mesmos. Por conseguinte, bens que antes eram tidos como de consumo básico e tratados como commodities, transformam-se em produtos de luxo, consumidos cada vez mais por camadas de maior poder socioeconômico (Aaker, 2010; Blue, 2009; Zenker \& Jacobsen, 2015). Com a vasta difusão desse fenômeno na Europa, houve necessidade de regularização, atribuída pelo Acordo de Lisboa, em 1954, que apresentou um primeiro sistema de registro, controle e proteção das denominações de origem internacionais. Entretanto, mesmo com o acordo em vigor, cada país ainda era livre para definir os requisitos para fins de registro e proteção de suas IGs. É apenas em 1994 com o TRIPS que padrões acerca do reconhecimento, registro, exercício e proteção de IGs são definidos e universalizados para todos os países membros da Organização Mundial do Comércio (OMC), o que o torna o acordo vigente e mais importante internacionalmente desta esfera (Das, 2010; Polido, 2013).

Através do Acordo TRIPs, os benefícios das indicações geográficas foram disseminados para os mais de 150 países membros da OMC, possibilitando que essas nações agreguem valor aos seus produtos por meio da sinalização de sua procedência (Barjolle, Quinones-Ruiz, Bagal \& Comoe, 2017). Assim, da união de pequenos produtores visando proteger sua produção, origina-se um instrumento legal de proteção de propriedade intelectual, criação de valor e vantagem competitiva. Para alcançar esses atributos, os produtores agrícolas perceberam que o local de produção era a base para o fortalecimento do produto, fosse através de seus recursos naturais (terroir) ou humanos/produtivos (savoir-faire) (Charters, Spielmann \& Babin, 2017). Por isso, divulgar essas características poderia traduzir esse valor em preços mais altos e, consequentemente, um lucro maior e diferenciação (Eren \& Dirsehan, 2017).

Atualmente, percebe-se que fatores antes vistos como prejudiciais à produção em menor escala, como a diminuição de barreiras econômicas e globalização, podem ser aproveitadas pois menores produtores também têm acesso a um mercado consumidor maior, uma vez seu produto estando sob a proteção e distinção refletidos pela indicação geográfica. Portanto, a mesma pode ser a base para a construção de ativos intangíveis para a comunidade, assim como a proteção de sua cultura e tradição local (Barjolle et al., 2017).

Além disso, as indicações geográficas fornecem um contraponto aos efeitos homogeneizadores da globalização e podem atender ao mercado crescente, formado por consumidores com consciência socioambiental e interessados em produtos com outras características e sabores (Garavaglia \& Mariani, 2017).

Verifica-se que, através desses benefícios, alguns países podem alcançar melhorias econômicas e sociais através do uso de indicações geográficas, por serem uma estratégia alternativa de desenvolvimento econômico, que prioriza a autonomia local e amplos objetivos de melhoria em toda a comunidade (Wilkinson, Cerdan \& Dorigon, 2017).

Como exemplo de sucesso, a Colômbia e seu setor cafeeiro, que passou a ser reconhecido internacionalmente como o melhor do mundo. Esse exemplo, assim como outros no Brasil (no setor cafeeiro e viticultor) e Índia (chá Darjeeling, arroz Basmati), deixam claro a eficácia da indicação geográfica na melhoria dos níveis econômicos de regiões e países em desenvolvimento (Brenes \& Chattopadhyay, 2013; Carvalho, Paiva \& Vieira, 2016; Das, 2010). 
No entanto, as IGs são um tópico novo para os países em desenvolvimento, cuja prática é recente e pouco desenvolvida, pois enfrentam desafios e dificuldades na sua implementação e gestão (Dokuzlu, 2016). A próxima seção examina mais profundamente cada um desses possíveis desafios e riscos.

\section{Desafios e riscos na implementação e gestão de indicações geográficas}

Por maior que seja a diversidade natural e sociodemográfica de uma região produtora, atributo esse significativo para a produção de um bem singular e distinguível, o país que não possuir ambiente político-legal apto para regularizar e monitorar instrumentos como as indicações geográficas terá dificuldades em aproveitar seus benefícios.

Mascarenhas e Wilkinson (2014) acrescentam à necessidade de normas e leis regulatórias para as indicações geográficas, a importância de uma tríade de apoio para seu desenvolvimento interno, formada por: 1) associação dos stakeholders dentro da cadeia produtiva, principalmente os produtores; 2) esforços do setor ao qual a produção pertence, em promover e distinguir a indicação geográfica; e 3) o auxílio de entidades públicos, principalmente no suporte e subsídio dessas ações.

Por ser de cunho coletivo, o consenso entre produtores acerca do nível de qualidade, nível de produtos no mercado, canais de distribuição adotados, práticas produtivas, entre outros, pode gerar conflito em qualquer fase de desenvolvimento da indicação geográfica. Somando-se a isso, a fraca tradição em coletividade e conformidade em alguns países e regiões pode deixar margem para que as normas e diretrizes, estabelecidas em conjunto pelos produtores, não sejam fielmente seguidos, o que pode colocar em risco a reputação e nome do local. Ademais, a multiplicidade de parceiros pode tornar difícil o consenso e uniformidade no direcionamento das ações e gestão da IG (Fonzo \& Russo, 2015).

Como símbolo que incorpora características físicas e culturais de certo local e, por isso, tem o papel de sinalizar qualidade e credibilidade de forma a atenuar a assimetria de informação entre produtor e consumidor, as indicações geográficas também apresentam desafios no que se diz respeito a gestão de imagem, reputação e ações de marketing.
A partir daí, são necessários recursos e ações direcionadas para que as associações entre o produto e o local sejam criadas e a percepção do consumidor seja desenvolvida. Mais que isso, é necessário despertar no consumidor o reconhecimento da região e a importância do elo produto-local para o desenvolvimento da mesma. Consequentemente, tornar uma indicação geográfica conhecida envolve esforços similares aos de fazê-lo para uma marca, pois requer tempo, recursos e conformidade de ações. Outrossim, a formação e manutenção de reputação, ligada aos valores da região, pode ser facilmente deteriorada, caso não haja conformidade de todos os produtores com as regras e padrões estabelecidos (Luceri, Latusi \& Zerbini, 2016; Marcoz, 2016).

Em acréscimo, problemas de divergência de imagem entre indicação geográfica e o país podem consumir recursos ainda maiores para estabelecê-la. Para melhor entendimento, pode-se citar o exemplo brasileiro e seu setor cafeeiro. O Brasil é conhecido internacionalmente como grande produtor de café, priorizando a escala em detrimento da qualidade. Tal imagem fora cristalizada desde o início da exploração do grão no país. Como resultado, as atuais indicações geográficas brasileiras de cafés enfrentam grandes desafios no estabelecimento da imagem de seu produto (café de alta qualidade), justamente pelo fato de o café brasileiro em geral ser reconhecido pelo oposto, ou seja, um bem de escala ou commodity (Dinnie, 2016; Nierdele \& Gelain, 2013). As dificuldades relacionadas a diferenciação de imagem entre indicação geográfica e país de origem são ainda maiores quando as IGs exportam seus produtos para mercados internacionais, o que ocorre principalmente com as estabelecidas em países em desenvolvimento, pelo fato do mercado interno não absorver totalmente a produção (Mascarenhas \& Wilkinson, 2014; Polido, 2013).

Primeiro, têm-se o custo de registrá-la nos países em que se pretende operar, o que consome recursos e tempo. Segundo, podem incorrer também custos de fiscalização de possíveis fraudes e falsificações, principalmente em países cujo cenário político-legal é ineficiente, o que acarreta tanto perdas financeiras como de imagem/reputação. Para mais, os gastos com monitoramento internacional são maiores, uma vez que envolve diferentes agências externas de controle (Das, 2010; Yeung \& Kerr, 2010). 


\section{2}

Uma Análise Crítica do Papel e Importância Socioeconômica das Indicações Geográficas em Países em Desenvolvimento

A partir da revisão de literatura, pode-se compreender a importância das indicações geográficas como propiciadoras de desenvolvimento local, assim como suas funções, benefícios e riscos. Como o intuito do artigo é o aprofundamento da relação destas com os países em desenvolvimento, a busca e análise de trabalhos até então publicados foi feita, em duas bases de dados distintas, sendo mais detalhadas a seguir.

\section{Metodologia}

Nesta seção são apresentados os procedimentos metodológicos utilizados neste estudo. Logo, os aspectos relativos à abordagem, estratégia de pesquisa, procedimentos para coleta e análise dos artigos são especificados e detalhados.

Por ter a finalidade de compreender mais sobre o desenvolvimento e aplicação das indicações geográficas, principalmente em países em desenvolvimento, assim como encontrar lacunas teóricas acerca do tema, a pesquisa é classificada como exploratória, utilizando a abordagem qualitativa (revisão de literatura) para alcançar seus objetivos e responder à pergunta de pesquisa (Malhotra, 2011). No intuito de assegurar a qualidade do presente artigo, optou-se pela escolha de base de dados internacionais e amplamente conhecidas.
Sendo assim, foram utilizados os bancos de dados Scopus e Web of Science. Tendo em vista a novidade do tema, a combinação de duas grandes bases de dados permitiria o acesso a um acervo maior e, consequentemente, maior possibilidade de encontro de trabalhos que envolvam e relacionam os temas pretendidos.

Para a definição de strings de busca, utilizou-se como base as palavras-chave (incluindo sinônimos e palavras relacionadas) encontradas em artigos e periódicos que abordam tais temas. Assim, os termos relacionados a Indicação Geográfica foram definidos como: (a) geographical indication; (b) origin certification; (c) designation of origin. De modo a não gerar repetição de trabalhos que contenham mais de um desses strings, utilizou-se o operador "OR" das bases de dados escolhidas. Ademais, pelo fato de o presente artigo tratar da análise de trabalhos que relacionem as duas áreas (indicação geográfica e países em desenvolvimento), os termos anteriores foram ligados com as palavras (d) developing country; (e) emerging markets; (f) emerging country, através do operador "AND" das bases de dados usadas, restringindo apenas os estudos que abordem concomitantemente as duas áreas focadas. Para melhor visualização, a figura a seguir ilustra esse processo.

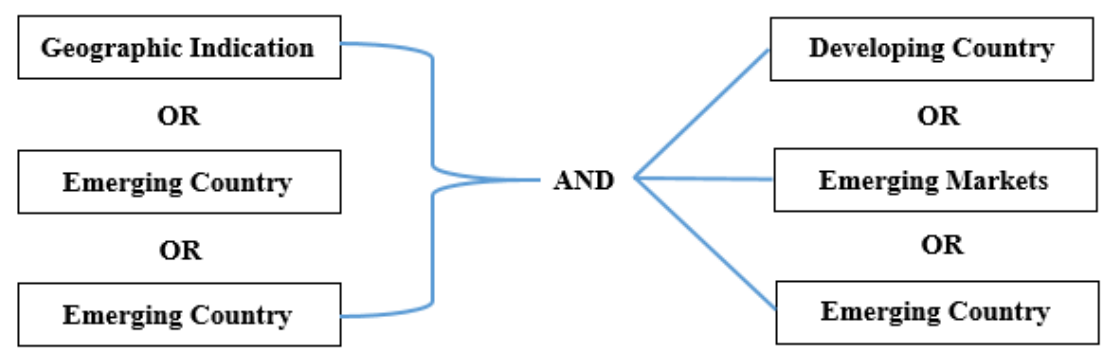

Figura 1: Protocolo de busca

Fonte: elaborado pelos autores.

Os termos foram buscados nos títulos, resumos e palavras-chave dos artigos, sendo excluídos os estudos duplicados e os não relacionados ao tema da pesquisa (Dias \& Mendes, 2018). Os artigos coletados são respectivos ao período de janeiro de 1998 a maio de 2018, intervalo esse que abrange a primeira publicação encontrada pela busca por termos e a data final em que essa etapa ocorreu.

A busca inicial através dos strings resultou em 42 artigos que, para que fossem compilados apenas os que se relacionassem aos temas, passaram pelos seguintes filtros, sequencialmente: i) leitura do título, resumo e palavras-chave; ii) leitura da introdução e conclusão. Assim, evidenciou-se que sete artigos não contemplavam o tema e, dessa forma, foram excluídos da análise. Logo, essa segunda etapa resultou em 35 artigos.

No intuito de tornar a base de artigos para análise ainda mais robusta, acrescentaram-se algumas das referências identificadas nesses 35 trabalhos, pela sua importância e relação com o tema. Em outras palavras, artigos não encontrados na busca inicial, 
mas que pudessem auxiliar na solução das questões de revisão anteriormente mencionadas, foram incluídos na base de artigos utilizando como filtro o título do artigo (Cooper, 2016). Essa terceira e última etapa do processo de busca e coleta (crossreferencing) acresceu à base de dados em 6 artigos, culminado no total final de 41 trabalhos. O período de busca e coleta de artigos pelos pesquisadores, incluindo suas três etapas (busca por termos, exclusão de artigos não relacionados ao tema e inclusão de artigos por meio de cross referencing) foi de maio a setembro de 2018.

Uma vez tendo formado uma base de análise com os artigos coletados, buscou-se encontrar similaridades entre eles no processo de leitura, principalmente nas perguntas e problemas de pesquisa propostos pelos autores, assim como nos resultados obtidos, para que os artigos pudessem ser organizados em categorias (Gaur \& Kumar, 2018). Assim, quatro esferas temáticas foram propostas como categorias para agrupá-los: político-legal, mercadológico, estratégia e estrutura interna. Os pesquisadores evitaram designar as categorias de maneira preconcebida, permitindo que seus nomes fluíssem dos artigos coletados (Kondracki, Wellman \& Amundson 2002), permitindo que as categorias e nomes das categorias fluam dos dados. As categorias auxiliaram os autores no reconhecimento e interpretação da conjuntura das IGs e seu desenvolvimento a partir de cada um desses pontos de vista (categorias) e, de modo a aumentar a validade do trabalho os autores leram e analisaram individualmente os artigos coletados e posteriormente discutiram os resultados e conclusões obtidos por cada um.

\section{Resultados}

Para melhor visualização, a Tabela 1 detalha os artigos encontrados, agrupando-os mais especificamente de acordo com: a origem do objeto de estudo, ou seja, região da indicação geográfica; o número de autores envolvidos; e o ano de publicação do artigo.

Tabela 1

Origem da IG, ano de publicação e quantidade de autores dos artigos

\begin{tabular}{|l|l|}
\hline Variável 1 - Origem das IGs & Número de Artigos \\
\hline Europa & 19 \\
\hline Ásia & 9 \\
\hline América do Sul & 8 \\
\hline África & 4 \\
\hline América do Norte & 1 \\
\hline Variável 2 - Número de Autores & \\
\hline 1 & 11 \\
\hline 2 & 19 \\
\hline 3 & 9 \\
\hline 4 & 2 \\
\hline Variável 3- Ano de Publicação & Número de Artigos \\
\hline 1998 a 2000 & 3 \\
\hline 2001 a 2010 & 10 \\
\hline 2011 ao presente & 28 \\
\hline
\end{tabular}

Fonte: elaborado pelos autores.

Pode-se perceber que as IGs de origem europeia são objeto de estudo da maioria das publicações, por já possuir certa tradição e histórico no tema, além de apresentar o maior número de registros. Como objeto de estudo, esses artigos analisam produtos para os quais a origem é um dos atributos que mais incorpora valor, como vinhos (Spielmann, 2015), queijos (Nacef, Lelievre-Demas, Drider, Flahaut \& 


\section{4}

Uma Análise Crítica do Papel e Importância Socioeconômica das Indicações Geográficas em Países em Desenvolvimento

Chollet, 2018) e azeites (Zanca et al., 2018), por exemplo. No entanto, vê-se o crescimento do interesse científico por objetos de estudo de outros continentes, como Ásia e América do Sul, visto que o fenômeno nesses locais é recente e, com base nesses trabalhos, mostra grande potencial econômico futuro, principalmente no setor agrário, predominante nesses países. Pelo fato de as IGs ainda estarem em processo de organização e legislação nos países emergentes (Dokuzlu, 2016; Mascarenhas \& Wilkinson, 2014), há a necessidade e interesse de compreensão de seu avanço nesses ambientes. De forma detalhada, as IGs investigadas são respectivas de, em ordem decrescente por país: França, Itália, Espanha, Grécia Bulgária (Europa); Índia, Turquia, China e Tailândia (Ásia); Colômbia, Brasil e México (América do Sul); Etiópia, Quênia e África do Sul (África); Estados Unidos (América do Norte).

Esses artigos, que têm como foco ou são originados nos países em desenvolvimento e que ainda não possuem uma base sólida para implantação de IGs, debatem os entraves e desafios que enfrentam, sejam eles de referência políticolegal (Neilson, Wright \& Aklimawati, 2018), de conflito e harmonia na formação e estrutura da indicação geográfica (Fonzo \& Russo, 2015), de falta de apoio nacional e externo assim como de escassez de recursos para monitorar e controlar fraudes e desvios (Barjolle et al., 2017; López-Bayón, GonzálesDías, Solís-Rodríguez \& Fernández-Barcala, 2018).

Outro ponto observado, além da novidade, é o gradativo interesse sobre o tema. Nota-se que, com o passar dos anos, as indicações geográficas passam a ser cada vez mais exploradas e pesquisadas pelos autores, particularmente após o Acordo sobre Aspectos dos Direitos de Propriedade Intelectual Relacionados ao Comércio (TRIPs - Agreement on Trade-Related Aspects of Intellectual Property Rights) de 1994 e o reconhecimento da IG como tipo de propriedade intelectual internacionalmente. 0 aumento gradativo de pesquisas sobre o tema e seu caráter inovador podem ser demonstrados na própria base de artigos coletados, como ilustrado na figura abaixo.

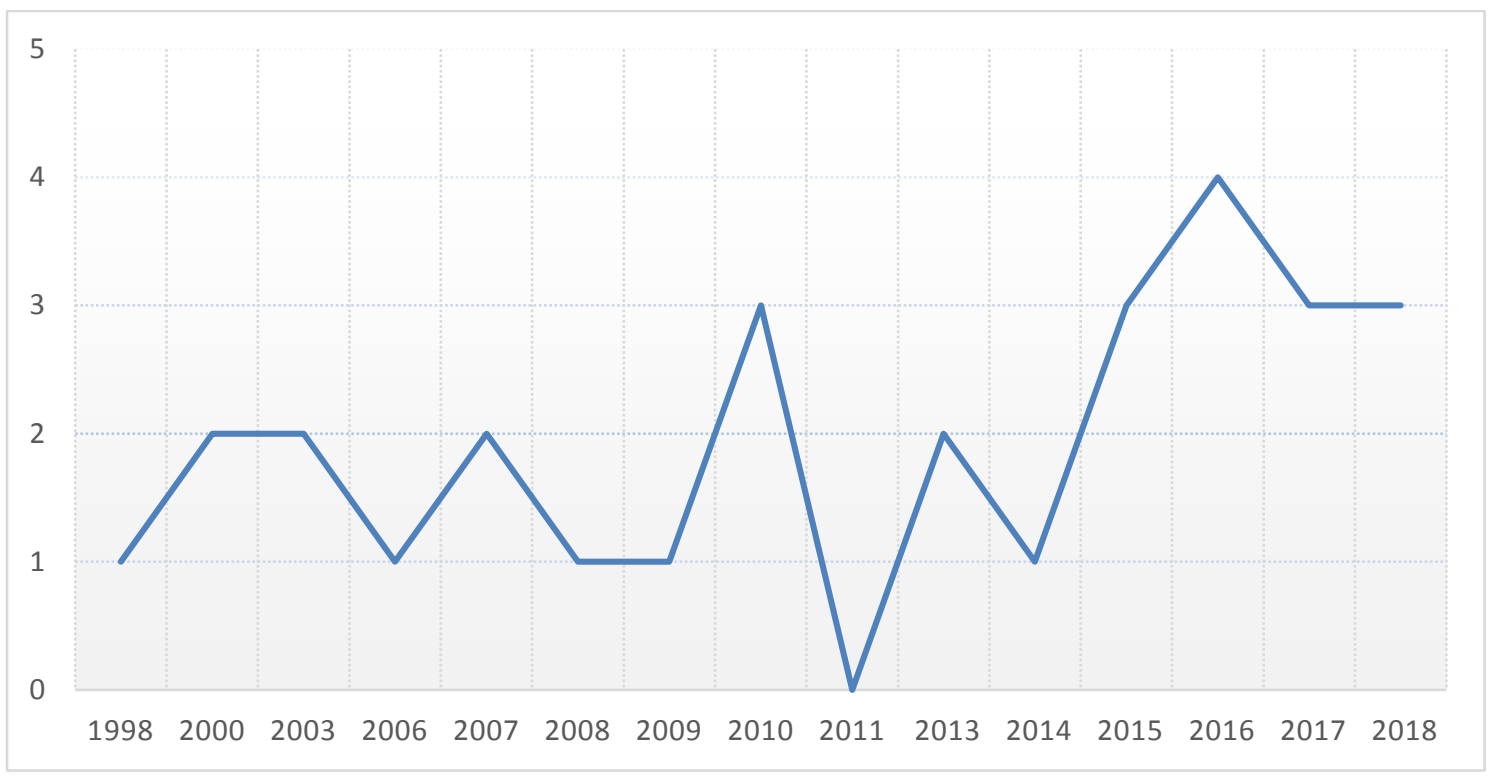

Figura 2: Distribuição anual das publicações

Fonte: elaborado pelos autores.

Com a leitura e análise crítica dos trabalhos, percebe-se que os pontos mais examinados a respeito das indicações geográficas podem ser agrupados em quatro frentes/enfoques diferentes, definidos neste artigo como: político-legal (instituições públicas participantes, leis e normas a respeito da obtenção e proteção da IG, tanto interna como externamente); (comportamento do consumidor, imagem e características do produto); estratégia (planejamento, gestão, objetivos e metas principais da IG); e estrutura interna (organização dos produtores, cooperativas, marca-coletiva). A Tabela 2 permite melhor visualização dos trabalhos analisados e qual das óticas estabelecidas e descritas acima fora utilizada para a abordagem dos artigos e trabalhos. 
Tabela 2

Número de artigos por foco

\begin{tabular}{|l|l|}
\hline Foco & Número de Artigos \\
\hline Político-Legal & 9 \\
\hline Mercadológico & 16 \\
\hline Estrutura e Organização & 7 \\
\hline Planejamento e Estratégia & 9 \\
\hline
\end{tabular}

Fonte: elaborado pelos autores.

Agrupados na categoria "político-legal", a semelhança desses artigos é o foco nas questões de legislação, infraestrutura institucional e registro de IGs e políticas de suporte e fiscalização.

Apesar do processo recente de legislação e jurisdição das indicações geográficas em vários países do mundo, assim como sua atuação internacional, poucos são os estudos que abordem as IGs pelas perspectivas legal, jurídica e de propriedade intelectual. Ainda, aqueles que examinam esse aspecto das IGs, têm como cerne de pesquisa produtos de países desenvolvidos, em sua maioria Europa, que possui aparato legal e jurídico consolidado para coordenação de IGs. Dos 41 trabalhos analisados, nove fornecem uma visão das questões institucionais e políticas no debate internacional sobre indicações geográficas.

Com relação aos diferentes produtos sob certificação de indicação geográfica estudados nos artigos buscados, ressalta-se que a maioria tem como foco produtos alimentares que se posicionam como de qualidade superior dentro desse segmento, como queijos, presuntos, azeites e vinhos de regiões específicas, já reconhecidas pelos consumidores. Essa última categoria de produto, principalmente, que possui periódicos próprios (International Journal of Wine Marketing e International Journal of Wine Business Research), foi o cerne de sete dos artigos encontrados. Em suma, 16 do total de artigos usam o ponto de vista econômico, relacionado ao comportamento do consumidor e a imagem emitida pela IG para formação de proposições e perguntas de pesquisas.

A maioria desses trabalhos apresenta como o centro das investigações o consumidor, características do produto e de imagem, sendo contidos na esfera denominada "mercadológica". Tenta-se identificar, por exemplo: os níveis de aceitação e preferência dos diferentes tipos de consumidor por produtos com indicação geográfica e o nível de influência dessa quando outros sinais (cues) são apresentados (Sepúlveda, Chekmam, Maza \& Mancilla, 2016); se a origem ou nacionalidade do consumidor tem papel no peso que esse dá para aspectos do produto como região de origem, de produção e tipo de certificação envolvida (Velčovská \& Sadílek, 2015); o modo como o grau de familiaridade e experiência do consumidor influem em sua preferência e intenção de compra de um produto com certificação de origem (Eren-Erdoğmuş \& Dirsehan, 2017) o quanto este é influenciado por indicações geográficas frente a outros tipos de certificação (ex: fair trade, produto orgânico), marcas globais e instituições maiores (made-in EU) (Diamantopoulos, Herz \& Koschate-Fischer, 2017).

Outra parte das pesquisas categorizadas nessa esfera examinam quais atributos são valorizados pelo consumidor em um produto com algum tipo de certificação de origem e se o mesmo consegue identificar essas características através das pistas extrínsecas e intrínsecas desse produto. Nesse âmbito, são significativos os estudos de Sepúlveda et al. (2016) sobre as preferências dos consumidores colombianos em relação à origem e alguns atributos do café (orgânico, especial, de origem); o de Spielmann (2015), que examina as avaliações dos consumidores de produtos típicos e atípicos de certas origens; e o de Ingrassia et al. (2016) acerca dos atributos e variáveis valorizados pelo consumidor de laranjas certificadas e produzidas em Riberella, no sul da Itália, caracterizando assim suas preferências e como relacioná-las a esses atributos do produto.

A partir dos artigos dessa esfera, percebe-se uma forte busca pela compreensão acerca de como a informação de origem influencia a avaliação do consumidor. Ademais, pouco se sabe sobre a percepção do consumidor em relação aos selos e 
rótulos de IG e a forma de comunicação usada para informar a origem do produto (Luceri, Latusi \& Zerbini, 2016). De forma prática, é relevante identificar se há uma avaliação diferente do consumidor quando o produto é oriundo de uma região protegida e delimitada dentro do território de um país em desenvolvimento, e submetida a padrões e critérios produtivos capazes de assegurar as características esperadas no produto (Charters, Spielmann \& Babin, 2017).

As respostas a essas questões são de grande relevância para os países em desenvolvimento pois, através da compreensão do consumidor e das indicações geográficas, características diferentes das refletidas pela imagem do país de origem ou região do produto podem ser realçadas, incorporando assim valor ao produto e atenuando conotações negativas (Dinnie, 2016).

Exemplos como o café colombiano (Martinez, 2014; Reina, Silva, Samper \& Fernández, 2007), os tecidos indianos (Das, 2010), os vinhos africanos (Engelbrecht, 2014; Merwe \& Maree, 2016), mostram produtos de origem reconhecidos mundialmente pela sua qualidade, apesar de serem produzidos em países cuja imagem não é tão favorável, muitas vezes remetendo a problemas socioeconômicos do país, como o narcotráfico na Colômbia (Reina et al., 2007); população dividida em castas e superpopulação na Índia (Das, 2010); problemas sociais e ambientais na África (Zenker, 2015); o que fomenta conotações negativas e pode afetar a imagem e percepção de valor do produto de IG desses países.

Ademais, outra gama de artigos analisam as indicações geográficas pela sua ótica estrutural e de mercado, ou seja, seu poder de influência: nos mercados, a nível de padrões de qualidade, preço, consumo; em setores e atividades diferentes da de produção do produto protegido (ex: Torre, ArjonaFuentes e Amador-Hidalgo (2017) constataram ganhos no setor turístico da região de Andaluzia, na Espanha, com o desenvolvimento da produção local de azeite protegido por (G); no incentivo à produção agrícola, melhora da renda de agricultores, combate ao despovoamento de certas áreas e melhoria competitiva, através da cooperação e coletivismo entre os membros da indicação geográfica (Castro \& Giraldi, 2018). Esses artigos, sete em seu total, foram organizados sob a ótica de "estrutura e organização", como especificado na Tabela 2.

Incorporando parte do conteúdo já apresentado ao planejamento estratégico e gestão das indicações geográficas, nove dos 41 artigos as examinam através de uma perspectiva estratégica e, por isso, foram dispostos na esfera nomeada "planejamento e estratégia" por examinarem questões como: alinhamento dos objetivos pretendidos com a implementação da IG (ex: maior preço de venda, proteção) com o planejamento das instituições e produção, de modo a valorizar e proteger o produto (ex: método de produção, colheita, cooperativas, associações, grêmios) (López-Bayón et al., 2018); fortalecer a IG como saída para uma imagem de país que afeta a região e sua produção negativamente (Martínez, 2016); internacionalizar a IG, assim como o desenvolvimento de mercados domésticos e externos para seus produtos (Alonso, 2017; Barjolle et al., 2017). Os trabalhos dessa categoria evidenciam demonstram o desalinhamento entre as IGs já estabelecidas nesses países e o pouco aproveitamento das possibilidades e vantagens das IGs que sobressaem à proteção, figurando entre: incentivo de diversificação e incremento da produção de diversos setores; melhoria da renda dos produtores e fortificação de alianças coletivas; otimização das condições socioambientais das regiões; e a oferta de produtos de alta qualidade para os consumidores locais (Fonzo \& Russo, 2015; Castro \& Giraldi, 2018).

A maioria dos trabalhos encontrados é fruto de pesquisa empírica, de natureza descritiva. Quanto à abordagem, há um misto de pesquisas qualitativas e quantitativas, com predominância de uso de dados primários, coletados principalmente através de surveys e entrevistas. A maior parte dos artigos é originado de estudos transversais, ou seja, de uma análise do fenômeno em determinado momento, pontual. Em menor número, poucos artigos apresentaram pesquisas do tipo teórica, de natureza exploratória ou utilizando mais de uma abordagem. Pode-se notar também que é pequena a parcela de artigos com estudos longitudinais, ou seja, ao longo do tempo. Para uma visão geral, a Tabela 3 classifica os trabalhos encontrados a partir dos critérios acima discutidos, ou seja, sua natureza, objeto e objetivos de estudo, método de coleta e tipo de dados utilizados e evolução no tempo. 
Tabela 3

Classificação das pesquisas

\begin{tabular}{|l|l|l|}
\hline Classificação & Tipo de Pesquisa & Total de Artigos \\
\hline \multirow{4}{*}{ Quanto ao tipo } & Empírica & 32 \\
\cline { 2 - 3 } & Teórica & 9 \\
\hline \multirow{5}{*}{ Quanto à abordagem à natureza } & Descritiva & 28 \\
\cline { 2 - 3 } & Exploratória & 13 \\
\hline \multirow{5}{*}{ Quanto à coleta de dados } & Qualitativa & 18 \\
\cline { 2 - 3 } & Quantitativa & 16 \\
\cline { 2 - 3 } & Qualitativa e Quantitativa & 7 \\
\hline & Bibliográfica & 7 \\
\cline { 2 - 3 } & Documental & 2 \\
\cline { 2 - 3 } & Entrevistas & 11 \\
\cline { 2 - 3 } & Estudo de Caso & 6 \\
\cline { 2 - 3 } & Experimento & 5 \\
\cline { 2 - 3 } & Survey & 16 \\
\hline \multirow{5}{*}{ Quanto ao tipo de dados } & Primários & 21 \\
\cline { 2 - 3 } & Secundários & 14 \\
\cline { 2 - 3 } & Mistos & 6 \\
\hline & Longitudinal & 30 \\
\cline { 2 - 3 } & Teórico & \\
\cline { 2 - 3 } & Transversal & 7 \\
\hline & & \\
\hline
\end{tabular}

Fonte: elaborado pelos autores.

\section{Discussões}

Por meio da busca e análise dos artigos, nota-se que o esforço científico em investigar as IGs no contexto e condições de países em desenvolvimento se mostra escasso. Portanto, para que as IGs possam ser utilizadas como ferramentas para melhorar índices socioeconômicos, é necessário que sua implementação e uso sejam explorados e compreendidos também em ambientes subdesenvolvidos e em desenvolvimento, de realidade aposta àqueles que já alcançaram níveis econômicos e sociais superiores, como a União Europeia, que é o foco da maioria dos artigos que analisam assuntos relacionados às IGs.

Com base na divisão proposta pelo presente artigo e explicitada na Tabela 2, a começar pelos trabalhos que focam principalmente no aspecto "político-legal" das IGs, busca-se compreensão e melhorias a respeito da estruturação legal e fiscal que os países emergentes têm desenvolvido para comportar suas indicações geográficas e empregá-las tanto interna como externamente. $O$ fato de grande parte dos artigos abordar a IG como propriedade intelectual e ser publicada em revistas desse gênero, como no caso das recentes pesquisas de Basole (2015), Wilkinson, Cerdan e Dorigon (2015), Belletti, Marescotti e Touzard (2017), corrobora essa elucidação.

O pobre desempenho de algumas das IGs pode ser explicado em termos de incapacidade dos ambientes institucionais locais dos países emergentes de apoiar suas IGs, principalmente na fase de implementação, estruturação e associação aos principais agentes do setor no qual estão inseridas, como indústrias, empresas, traders e outras associações de produtores, por exemplo. Percebe-se também que, embora uma das intenções das IGs seja afirmar a reivindicação moral sobre a propriedade geográfica e cultural incorporada aos produtos, elas exigem um envolvimento muito maior em questões extra-legais 


\section{8}

Uma Análise Crítica do Papel e Importância Socioeconômica das Indicações Geográficas em Países em Desenvolvimento

ao longo da cadeia de valor para alcançar o estimado desenvolvimento regional.

A partir dessa percepção, pode-se concluir que esses países ainda estão em estágio prematuro no que se diz respeito às IGs, uma vez que a instituição e legitimação da ferramenta é o cerne das pesquisas dessa categoria, diferente de países europeus e norte-americanos que já possuem aparato legal desenvolvido, órgãos especializados e experiência para lidar com dilemas relacionados aos aspectos legais das IGs, além de apresentarem outras questões e investigações em seus estudos. A despeito dos acordos internacionais, em especial o TRIPS, as nações possuem liberdade na gestão e coordenação das IGs internamente (Fonzo \& Russo, 2015). Pesquisas nessas áreas, em especial exploratórias, demonstram alta probabilidade de que as instituições desses países ainda não entraram em consenso acerca do tema e que a legislação e diretrizes dessas nações ainda não adequaram processos para legitimar as IGs e instruir produtores de sua natureza e funcionamento (López-Bayón et al., 2018).

Nesse ponto, não só tempo é essencial para galgar evolução e elaboração jurídico legal, mas também recursos que se destinem à proteção, monitoramento e fiscalização das indicações geográficas nos países em desenvolvimento. Mais do que estabelecimento de normas e proteção contra má utilização e falsificações, os recursos destinados a esses fins são também encarregados, mesmo que indiretamente, à manutenção da reputação e imagem refletida pela IG, o que pode ser primordial para os países emergentes destacarem seus produtos (Carvalho, Paiva \& Vieira, 2016). Apesar dessa necessidade de incentivo e políticas públicas para que as IGs possam se desenvolver e se preservar, são mínimos os investimentos feitos pelos países emergentes nesse sentido, o que pode culminar em ações de concorrentes e free-riders que venham a deteriorar a credibilidade e imagem da IG (Luceri, Latusi \& Zerbini, 2016). Outrossim, a formação e manutenção de reputação, ligada aos valores da região, pode ser facilmente danificada, caso não haja consonância entre todos os produtores com as regras e padrões estabelecidos (Charters, Spielmann \& Babin, 2017).

Em relação à divisão "mercadológica", como as indicações geográficas dos países emergentes compõem setores do agronegócio, produtores podem enfrentar problemas de imagem quando o país possui notoriedade em características contrastantes com as promovidas pela IG (Dinnie, 2016). Para atenuar tal contradição, planos e ações de marketing mais direcionados e alinhados com a identidade e objetivos da IG precisam ser elaborados, contando com o auxílio de apoio público, especialistas na área, universidades, agências de pesquisas e instituições governamentais. Aliada à reputação, a imagem da indicação geográfica, assim como a marca de um produto, é o símbolo do vínculo entre o local de produção e o bem produzido e, portanto, precisa ser fortalecido para que o consumidor reconheça a IG e o valor que concede ao produto (Aaker, 2010; Josias, Frikkie \& Bruwer, 2014). Contudo, muitos produtores de alimentos discutem se um maior uso da sinalização das IGs no produto seria uma forma de promovê-lo melhor, quando se tratando de commodities (Grunert \& Aachmann, 2016).

Nota-se também que o consumo de produtos certificados e de origem ainda se mostra em fase inicial nesses países, pois normalmente são comercializados a preços mais altos e, portanto, restritos a poucas camadas sociais da população (Neilson, Wright \& Aklimawati, 2018). Logo, os trabalhos que tratam desse tema analisam e caracterizam o público-alvo, no intuito de identificar o que o mesmo percebe como valor e como a comunidade/região pode estruturar sua produção, direcionando esforços para compor características que sejam essenciais ao produto, concomitante ao equilíbrio de preços (Dias \& Mendes, 2018). Ademais, aspectos como familiaridade, envolvimento e experiência são relacionados com a importância atribuída pelos consumidores às certificações de origem, com a finalidade de afunilar ainda mais o público-alvo (Giacalone, Fosgaard, Steen \& Münchow, 2016; Martínez, 2016).

No entanto, constata-se que grande parte desses estudos é feita de forma pontual, o que resulta em deduções e inferências limitadas, focadas em públicos e produtos específicos, pois respondem a questionamentos respectivos a faceta econômica e de mercado de uma região, e não ao contexto e aplicação da IG como um todo. Ou seja, é fundamental que questões mercadológicas mais amplas a respeito das IGs e sua aplicação sejam estudadas nos cenários de países emergentes, como por exemplo: se a origem do produto é realmente importante para o consumidor desses países; como e quanto a indicação geográfica de um produto 
influencia na compra, em comparação a outras características de produto (ex: preço, embalagem, propaganda, entre outros.); se possui o mesmo poder de influência quando equiparada a outros signos (marcas globais ou endossantes, por exemplo); educação e preparação do consumidor para o consumo do bem. Com o esclarecimento dessas questões, os países emergentes poderão direcionar melhor seus esforços para estruturar e aprimorar a imagem de sua(s) IG(s); entender melhor a percepção de seu consumidor e comunicar de maneira eficiente a IG e seu produto; adequar o valor esperado ao entregue; criar reconhecimento acerca da IG e seu produto; entre outros. Essas lacunas, quando preenchidas, trarão competitividade para os produtos com IG desses países, melhorando seu desempenho no mercado interno e externo (ao competir, por exemplo, com marcas e IGs consolidadas de países desenvolvidos).

Olhando-se pela perspectiva de "planejamento e estratégia" que alguns artigos investigam nas indicações geográficas, essas são definidas como plataformas para geração de vantagem competitiva, pois envolvem um produto com características distintas e difíceis de copiar, por serem resultado de um determinado local e interações produtivas (Charters, Spielmann \& Babin, 2017). Somando-se a isso, constata-se que a teoria avança na busca por modos de expansão da comercialização dos produtos protegidos por indicação geográfica, na busca de maiores ganhos, principalmente internacionalmente, uma vez que o mercado interno dos países emergentes possa não apresentar o potencial necessário para absorvê-los.

Esse crescimento pode ser facilitado por alianças estratégicas entre partes interessadas, tanto privadas quanto públicas, verticais ou horizontais. A cooperação entre os produtores em torno da indicação geográfica, sua estruturação e formação de identidade são assuntos tratados pelos artigos contidos na esfera "estrutura e organização". Nesse ponto, pode-se concluir que, nos países emergentes, as dificuldades se dão na própria estruturação da IG e no estabelecimento de cooperação e consenso entre os seus membros. Tais questões podem atravancar a formação de um grupo harmônico, do padrão de confecção necessário para manter as características dos produtos ao longo das produções, e de uma identidade consistente. Há também dificuldades no compartilhamento dos gastos e custos do estabelecimento da IG, ou seja, os recursos e investimentos necessários para sua legalização, comercialização, comunicação e proteção. Em suma, o maior desafio apontado é o da união, equilíbrio e cooperação em contraposição à competição entre os membros da IG, sobretudo para os países emergentes. Uma vez que as IGs surgem e se mantêm no coletivo, rivalidades e disputas infrutíferas entre os membros pode prejudicar o propósito da IG: valorização e proteção do produto local.

Outros aspectos averiguados na maioria dos artigos destacam o impacto que as IGs podem apresentar, por exemplo, sua capacidade transformadora na comunidade/local produtor; e o incremento de outras atividades econômicas e sociais, como turismo, agricultura e abastecimento, meio ambiente e sustentabilidade e inovação. Ou seja, os artigos auxiliam a compor a conclusão do papel modificador e agregador de valor que as indicações geográficas trazem para os locais, seus produtos e produtores, sendo implementada numa variedade extensa de diferentes categorias de produto, setores e países. Logo, a leitura crítica dos artigos corrobora com o fato de que as indicações geográficas, além de ferramentas protetoras e diferenciadoras de produto, podem galgar desenvolvimento e melhores condições de mercado e competição. Mesmo assim, as potencialidades da IG são pouco exploradas em países em desenvolvimento e enfrentam grandes obstáculos em sua implementação, gestão e fiscalização, justamente pelo ambiente legal, jurídico e fiscal desses países.

Por se tratar de um estudo qualitativo, a primeira limitação está ligada às interpretações dos autores, pois variam em profundidade e nível de abstração, dependendo do método de análise e da capacidade de se distanciar do objeto estudado.

Outro fator limitante é o tamanho da amostra. O total de 41 artigos analisados prevê cautela na generalização de seus resultados. Em adição a esse ponto, a busca de artigos não considerou aqueles redigidos em línguas que não o inglês, o que pode ter deixado de fora artigos específicos sobre IGs em países da América Latina ou Ásia, por exemplo. Por conseguinte, os estudos encontrados podem não tratar de parte considerável das IGs de países em desenvolvimento, o que acarreta certo viés para as interpretações e discussões dos autores.

Pelo fato de a base de artigos constituir IGs de países extremamente diferentes em questões sociopolíticas, econômicas, culturais e ambientais, a 
Uma Análise Crítica do Papel e Importância Socioeconômica das Indicações Geográficas em Países em Desenvolvimento

visão e a conduta que os consumidores locais e os governos possuem do mesmo tema também se diferem, o que pode interferir nas inferências apresentadas no trabalho.

\section{Conclusões e Sugestões para Pesquisas Futuras}

O objetivo deste artigo foi compreender o papel e a importância das indicações geográficas como possíveis ferramentas de alavancagem de desenvolvimento socioeconômico, principalmente para regiões e países em desenvolvimento. Para alcançar esse propósito, os objetivos específicos foram constituídos de modo a compreender como as regiões e países, principalmente em desenvolvimento, podem ser impactados pelas indicações geográficas. Como possíveis desafios, a análise dos trabalhos coletados resultou em fatores como: a falta de preparo institucional e legal dos países emergentes, que ainda não estão prontos para apoiar e defender tais iniciativas; a dificuldade de promover a IG em contraponto com a imagem negativa do país de origem; os entraves em alcançar harmonia na estruturação e organização dos produtores ante a IG; e a falta de estipulação de metas comuns, que beneficiem a todos, além do planejamento coletivo para alcançá-las.

No entanto, as vantagens e contribuições que as IGs podem trazer para os países emergentes superam essas possíveis dificuldades, acarretadas pela sua implementação e manutenção, sendo muitas as ocasiões nas quais sua utilização transformaram a realidade socioeconômica das comunidades envolvidas. Para tanto, os casos apresentados pela literatura mostram que os esforços dos produtores em manter constância, harmonia e consistência no planejamento e gestão da IG, somados às ações públicas de suporte e capacitação da comunidade, além de formação de um aparato jurídico-legal de sustentação da ferramenta, são pontos essenciais para a formação de uma IG eminente.

Além da contribuição prática, a pesquisa dispõe um panorama teórico atual sobre o tema, evidenciando que, apesar das potencialidades das indicações geográficas como ferramentas de alavancagem de desenvolvimento, ainda é pequena a exploração e aprofundamento da área em termos científicos.

Logo, como possibilidade de estudos futuros, sugere-se que outras variáveis relacionadas às IGs sejam investigadas, ou seja, a relação dessas com alterações em indicadores como: nível de desemprego, poder de compra da comunidade, educação e investimentos, entre outros (Mascarenhas \& Wilkinson, 2014). As diferenças metodológicas empregadas nos artigos disponíveis levam a resultados variados e, por isso, impossibilitam o rastreamento preciso do desenvolvimento do tema ao longo do tempo. Por isso, é importante que estudos futuros empreguem a mesma metodologia, aplicada longitudinalmente, para melhorar a compreensão sobre o desenvolvimento das IGs em países emergentes.

É incontestável que as instituições públicas influenciam o processo de evolução da IG sendo, portanto, necessária a compreensão do papel das mesmas na criação, estruturação e gestão de IGs (Basolle, 2015; Barjolle et al., 2017; Wilkinson et al., 2017). Somando-se a isso, torna-se fundamental estudos que auxiliem no planejamento e execução estratégica de indicações geográficas, obtendo sucesso no aproveitamento das vantagens naturais e humanas que a região oferece (Yeung \& Kerr, 2011; Charters, Spielmann \& Babin, 2017).

Ademais, conclui-se através dos artigos que focam em países em desenvolvimento que, muitas vezes, a imagem do país pode atrapalhar o crescimento e reputação da IG. Sendo assim, torna-se importante que as próximas pesquisas explorem maneiras para atenuar a imagem negativa do país para determinado setor, de modo que a IG possa se sobressair e criar uma percepção positiva nos consumidores internos e externos (Diamantopoulos, Herz \& Koschate-Fischer, 2017; Likoudis, Sraldi, Costarelli \& Apostolopoulos, 2016). Assim como o estudo da imagem, o entendimento do consumidor ou público alvo interno e externo das indicações geográficas é essencial, assim como o que é percebido como valor e quais são os fatores que mais os influenciam, sendo, portanto, outra recomendação para os próximos trabalhos (Eren \& Dirseban, 2017).

Por fim, é de suma pertinência que estudos focalizem na compreensão dos meios de disseminação de fidelidade/lealdade de um produto existente para outros produzidos na mesma região ou para outros de locais próximos (Charters, Spielmann \& Babin, 2017), além da identificação e esquematização de mecanismos de co-criação de valor que envolvam as partes interessadas de IGs diferentes, em conjunto com suas respectivas cidades, regiões ou países (Martínez, 2016). 


\section{Referências}

Aaker, D. (2010) Building Strong Brands. London: Simon \& Schuster.

Alonso, A. D. (2017) An exploration of Cava wineries: a resource-based approach. International Journal of Wine Business Research, 29 (1): 20-36.

Barjolle, D., Quinones-Ruiz, X. F., Bagal, M., \& Comoe, H. (2017) The role of the state for geographical indications of coffee: case studies from Colombia and Kenya. World Development, 98: 105-119.

Basole, A. (2015) Authenticity, innovation, and the geographical indication in an artisanal industry: the case of the Banarasi Sari. The Journal of World Intellectual Property, 18 (3-4):127 149.

Blue, G. (2009) Branding beef: marketing, food safety, and the governance of risk. Canadian Journal of Communication, 34: 229-244.

Bowen, S. (2010) Development from within? The potential for geographical indications in the global south. The Journal of World Intellectual Property, 13 (2): 231-252.

Brenes, E. R., Chattopadhyay, A., \& Montoya, D. (2013) Grupo Britt N.V.: should it seek to expand business in the USA? Academia Revista Latinoamericana de Administración, 26 (3): 373-394.

Carvalho, J. M., Paiva, E. L., \& Vieira, L. (2016) Quality attributes of a high specification product: evidences from the speciality coffee business. British Food Journal, 118 (1): 132-149.

Castro, V. A., \& Giraldi, J. M. E. (2018) Shared brands and sustainable competitive advantage in the Brazilian wine sector. International Journal of Wine Business Research, 30 (2): 243-259.

Chamorro, A., Rubio, S., \& Miranda, J. (2015) The region-of-origin (ROO) effect on purchasing preferences: the case of a multiregional designation of origin. British Food Journal, 117 (2): 820-839.

Charters, S., Spielmann, N., \& Babin, B. J. (2017) The nature and value of terroir products. European Journal of Marketing, 51 (4): 748-771.

Clarke, I., Owens, M., \& Ford, J. B. (2000) Integrating country of origin into global marketing strategy: a review of US marking statues. International Marketing Review, 17 (2): 114-126.

Cooper, D. R., \& Schindler, P. S. (2016) Métodos de Pesquisa em Administração. 12nd ed. New York: McGraw-Hill.
Das, K. (2010) Prospects and challenges of geographical indications in India. The Journal of World Intellectual Property, 13 (2): 148-201.

Diamantopoulos, A., Herz, M., \& Koschate-Fischer, N. (2017) The EU as superordinate brand origin: an entitativity perspective. International Marketing Review, 34 (2): 183-205.

Dias, C., \& Mendes, L. (2018) Protected Designation of Origin (PDO), Protected Geographical Indication (PGI) and Traditional Speciality Guaranteed (TSG): a bibiliometric analysis. Food Research International, 103: 492-508.

Dimara, E., \& Skuras, D. (2003) Consumer evaluations of product certification, geographic association and traceability in Greece. European Journal of Marketing, 37 (5): 690-705.

Dinnie, K. (2016) Nation Branding: Concepts, Issues, Practice. 2nd ed. Abingdon: Routledge.

Dokuzlu, S. (2016) Geographical indications, implementation and traceability: Gemlik table olives. British Food Journal, 118 (9): 2074-2085.

Duguid, P. (2005) Networks and knowledge: the beginning and end of the Port Commodity Chain, 17031860. The Business History Review, 79 (3): 493-526.

Elango, B., \& Wieland, J. R. (2017) Impact of multilevel boundaries on internationalization: an exposition of region of origin effect, Multinational Business Review, 25 (3): 206-221.

Engelbrecht, J., Herbst, F., \& Bruwer, J. (2014) Region-of-origin (ROO) certification as marketing strategy in the South African wine market. International Journal of Wine Business Research, 26 (2): 139-162.

Eren-Erdogmus, I.., \& Dirsehan, T. (2017) Exploring local vs global brand associations in an emerging market using BCM technique. Qualitative Market Research: An International Journal, 20 (3): 266-288.

Fonzo, A., \& Russo, C. (2015) Designing geographical indication institutions when stakeholders' incentives are not perfectly aligned. British Food Journal, 117 (10): 2484-2500.

Fotopoulos, C., \& Krystallis, A. (2003) Quality labels as a marketing advantage: the case of the PDO Zagora apples in the Greek market. European Journal of Marketing, 37 (10): 1350-1374.

Giacalone, D., Fosgaard, T. R., Steen, I., \& Münchow, M. (2016) Quality does not sell itself": divergence between "objective" product quality and preference for 


\section{2}

Uma Análise Crítica do Papel e Importância Socioeconômica das Indicações Geográficas em Países em Desenvolvimento

coffee in naïve consumers. British Food Journal, 118 (10): 2462-2474.

Grunert, K. G., \& Aachmann, K. (2016) Consumer reactions to the use of EU quality labels on food products: a review of the literature. Food Control, 59: 178-184.

Herrera, C. F., \& Blanco, C. F. (2011) Consequences of consumer trust in PDO food products: the role of familiarity. Journal of Product \& Brand Management, 20 (4): 282-296.

Ilbert, H., \& Petit, M. (2009) Are geographical indications a valid property right? Global trends and challenges. Development Policy Review, 27 (5): 503-528.

Josias, A. E., Frikkie, H., \& Bruwer, J. (2014) Regionof-origin (ROO) certification as marketing strategy in the South African wine market. International Journal of Wine Business Research, 26 (2): 139-162.

Kondracki, N., Wellman, N. S., \& Amundson, D. R. (2002) Content analysis: review of methods and their applications in nutrition education. Journal of Nutrition Education and Behavior, 34 (4): 224-230.

Lerdthamtewe, P. (2014) The protection of geographical indications in Thailand. World Intellectual Property, 17 (3-4): 114-128.

Likoudis, Z., Sradli, D., Costarelli, V., \& Apostolopoulos, C. (2016) Consumers' intention to buy protected designation of origin and protected geographical indication foodstuffs: the case of Greece. International Journal of Consumer Studies, 40: 283-289.

Luceri, B., Latusi, S., \& Zerbini, C. (2016) Product versus region of origin: which wins in consumer persuasion? British Food Journal, 118 (9): 2157-2170.

Malhotra, N. K. (2011). Pesquisa de Marketing: uma orientação aplicada. 6. ed. Porto Alegre: Bookman.

Malorgio, G., \& Grazia, C. (2007) Quantity and quality regulation in the wine sector: the Chianti Classico appellation of origin. International Journal of Wine Business Research, 19 (4): 298-310.

Marie-Vivien, D. (2008) From plant variety definition to geographical indication protection: a search for the link between basmati rice and India/Pakistan. World Intellectual Property, 11 (4): 321-344.

Mascarenhas, G., \& Wilkinson, J. (2014) Indicações geográficas em países em desenvolvimento: potencialidades e desafios. Revista de Política Agrícola, 23 (2): 103-115.

Martínez, N. M. (2016) Towards a network place branding through multiple stakeholders and based on cultural identities: the case of "The Coffee Cultural Landscape" in Colombia. Journal of Place Management and Development, 9 (1): 73-90.

Merwe, A., \& Maree, T. (2016) The behavioural intentions of specialty coffee consumers in South Africa. International Journal of Consumer Studies, 40: 501-508.

Nacef, M., Lelievre-Demas, M., Drider, D., Flahaut, C., \& Chollet, S. (2018) Artisanal and industrial Maroilles cheeses: are they different? Comparison using sensory, physico-chemical and microbiological approaches. International Dairy Journal, 89: 42-52.

Neilson, J., Wright, J., \& Aklimawati, L. (2018) Geographical indications and value capture in the Indonesia coffee sector. Journal of Rural Studies, 59: 3548.

Nierdele, P. A., \& Gelain, J. (2013) Geographical indications in Brazilian food markets: quality conventions, institutionalization and path dependence. Journal of Rural Social Sciences, 28 (1): 26-53.

Polido, F. B. P. (2013). Direito Internacional da Propriedade Intelectual: Fundamentos, Princípios e Desafios. Rio de Janeiro: Renovar.

Raustiala, K., \& Munzer, S. R. (2007) The global struggle over geographic indications. The European Journal of International Law, 18 (2): 337-365.

Reina, M., Silva, G., Samper, L. F., \& Fernández, M. P. (2007). Juan Váldez: La Estrategia Detrás de la Marca. Colômbia: Cargraphics S.A.

Sepúlveda, W. S., Chekmam, L., Maza, M. T., \& Mancilla, N. O. (2016) Consumers' preference for the origin and quality attributes associated with production of specialty coffees: results from a cross-cultural study. Food Research International, 89: 997-1003.

Spielmann, N. (2015) Anything but typical: how consumers evaluate origin products based on their cues. International Journal of Wine Business Research, 27 (1): 23-39.

Terziyska, I. (2017) Benchmarking wine tourism destinations: the case of Bulgaria. International Journal of Wine Business Research, 29 (4): 384-400.

Thode, S. F., \& Maskulka, J. M. (1994) Place-based marketing strategies, brand equity and vineyard valuation. Journal of Product \& Brand Management, 7 (5): 379-399.

Velčovska, Š., \& Sadílek, T. (2015) Certification of cheeses and cheese products origin by EU countries. British Food Journal, 117 (7): 1843-1858. 
Vertinsky, I., \& Zhou, D. (2000) Product and process certification: systems, regulations and international marketing strategies. International Marketing Review, 17 (3): 231-253.

Viot, C., \& Passebois-Ducros, J. (2010) Wine brands or branded wines? The specificity of the French market in terms of the brand. International Journal of Wine Business Research, 22 (4): 406-422.

Wargenau, A., \& Che, D. (2006) Wine tourism development and marketing strategies in Southwest Michigan. International Journal of Wine Marketing, 18 (1): 45-60.

Wilkinson, J., Cerdan, C., \& Dorigon, C. (2017) Geographical indications and "origin" products in Brazil: the interplay of institutions and networks. World Development, 98: 82-92.

World Intellectual Property Organization Geographical Indications. Recuperado

(2018) https://www.wipo.int/geo_indications/en/.
WIPO (2019), “Geographical indications world intellectual property organization", available at: www.wipo.int/geo_indications/en/ (accessed 29 May 2019).

Yeung, M. T., \& Kerr, W. A. (2011) Are geographical indications a wise strategy for developing country farmers? Greenfields, clawbacks and monopoly rents. The Journal of World Intellectual Property, 14 (4): 353367.

Zanca, A. T., Santos, R. A., Mejias, M. L., Izquierdo, M., Cancilla, J. C., \& Torrecilla, J. S. (2018) Laser diode induced excitation of PDO extra virgin olive oils for cognitive authentication and fraud detection. Sensors \& Actuators B. Chemical, 290: 1-9.

Zenker, S. \& Jacobsen, B. P. (2015). Inter-Regional Place Branding: Best Practices, Challenges and Solutions. New York: Springer International Publishing.

\section{Sobre os Autores}

- Mateus Manfrin Artêncio - Faculdade de Economia, Administração e Contabilidade de Ribeirão Preto - FEARP/USP, São Paulo, (Brasil). E-mail: mateus.manfrin@gmail.com Orcid id: https://orcid.org/0000-00024875-2063.

- Janaina de Moura Engracia Giraldi - Faculdade de Economia, Administração e Contabilidade de Ribeirão Preto - FEA-RP/USP, São Paulo, (Brasil). E-mail: jgiraldi@usp.br Orcid id: https://orcid.org/0000-0001-7150$\underline{2217}$.

- $\quad$ Simone Vasconcelos Ribeiro Galina - Faculdade de Economia, Administração e Contabilidade de Ribeirão Preto - FEA-RP/USP, São Paulo, (Brasil). E-mail: svgalina@usp.br Orcid id: https://orcid.org/0000-00017150-2217. 


\title{
A CRITICAL ANALYSIS OF SOCIOECONOMIC IMPORTANCE AND ROLE OF GEOGRAPHICAL INDICATIONS IN DEVELOPING COUNTRIES
}

\author{
Mateus Manfrin Artêncio, Janaina de Moura Engracia Giraldi, \\ Simone Vasconcelos Ribeiro Galina \\ Faculdade de Economia, Administração e Contabilidade de Ribeirão Preto - FEA-RP/USP, São Paulo, (Brasil).
}

\begin{tabular}{l} 
ARTICLE DETAILS \\
\hline \\
Article history: \\
Received: 10 December 2018 \\
Accepted: 08 June 2019 \\
Available online August: 01 th 2019 \\
Double Blind Review System \\
Scientific Editor \\
Ilan Avrichir
\end{tabular}

\section{Keywords}

Origin Information Geographical Indication

Developing countries

\begin{abstract}
The article brings a perspective of the role and importance of geographical indications (GIs) in developing and emerging countries. Gls became potential tools capable of enhancing local socioeconomic indices, through the protection and valorization of the product and region itself. Therefore, academic papers that addressed the implementation and management of GIs in countries with different levels of development were analyzed in a systematic angle. The articles were researched and collected with the help of Scopus and Web of Science databases. The research is classified as exploratory and bibliographical, based on critical reading of 40 articles. Among them, some demonstrate the process of implementation and development of Gls, inasmuch as the challenges they face. Others analyze the internal structural issues and strategy planning of GIs as a collective arrangement. Another portion measures and analyzes the image that a GI transmits as a brand to consumers. From this analysis, it was verified that none of the articles specifically relates or examines geographical indication capability as a development leverage tool for developing countries / regions, finding a gap in the theoretical framework of the area and the need for studies and practical cases that demonstrate this relationship, since many regions benefit from that
\end{abstract}

Para citar este artigo:

Manfrin Artêncio, M., de Moura Engracia Giraldi, J., \& Vasconcelos Ribeiro Galina, S. (2019). Uma Análise Crítica do Papel e Importância Socioeconômica das Indicações Geográficas em Países em Desenvolvimento. Internext, 14(3), 218 -234. doi:http://dx.doi.org/10.18568/internext.v14i3.483 